\title{
Histopathological study of placentae in intrauterine growth retardation pregnancies in a tertiary care hospital and correlation with fetal birth weight
}

\author{
Mardi Kavita ${ }^{1}$, Negi Lalita ${ }^{1}$ \\ ${ }^{\prime}$ Deptartment of pathology, Indira Gandhi Medical College, Shimla, India
}

\author{
Keywords: \\ Birth weight; \\ Chorionic villi; \\ Cytotrophoblasts; \\ Fetus; \\ IUGR; \\ Placenta; \\ Syncytial knotting
}

\begin{abstract}
Background: Intra uterine Growth Retardation is the most significant factor of perinatal mortality. The aim of this study was to assess the histopathological changes in the placenta in association with IUGR and correlation with fetal birth weight.
\end{abstract}

Materials and methods: A total of 100 placentae were included. Twenty five normal placentae and 75 placentae were from IUGR pregnancies were included.

Results: Intervillous fibrin deposition (64\%), increased syncytial knotting (64\%), stromal fibrosis (65\%), cytotrophoblastic hyperplasia (44\%) and basement membrane thickening (40\%) were seen along with hypovascular villi and infraction were present in $32 \%$ and $28 \%$ respectively. These changes were seen less in the control group $(\mathrm{p}<0.001)$. Statistically significant association between the birth weight and microscopic changes (chi square $=19.543$, degree of freedom $=4, p<0.005$ ) was observed.

Conclusion: Severity of intrauterine growth retardation is related to the microscopic change in the placenta. The number and severity of microscopic changes in IUGR placentas increased with decreasing fetal birth weight.

\section{Correspondence:}

Dr Kavita Mardi MD,DNB

Department of Pathology

Set No 14, Type VI Quarters, Department of Pathology, Indira Gandhi

Medical College, Shimla, India

ORCID ID: 0000-0001-8351-3554

Email: kavitamardi @yahoo.co.in

Reveived : April 2nd, 2017 ; Accepted : August 3rd, 2017; Published : September 1, 2017

Citation: Mardi K, Negi L. Histopathological study of placentae in intrauterine growth retardation pregnancies in a tertiary care hospital and correlation with fetal birth weight. J Pathol Nep 2017;7:

1176-9. doi: 10.3126/jpn.v7i2.18003

Copyright: This is an open-access article distributed under the terms of the Creative Commons Attribution 4.0 International License, which permits unrestricted use, distribution, and reproduction in any medium, provided the original author and source are credited.

\section{INTRODUCTION}

The antenatal health care given to pregnant women has great influence on the rates of perinatal death and morbidity. Amongst the different causes of perinatal mortality, Intra Uterine Growth Retardation (IUGR) is the single most significant factor and about $2 / 3$ deaths among infants with less than $2500 \mathrm{~g}$ birth weight. ${ }^{1}$ Causes of IUGR can be divided into maternal, fetal, placental and unknown causes, but the basic pathophysiology is due to reduced availability of nutrients in mother or its reduced transfer by the placenta to the fetus. It may also be due to reduced utilization by the fetus. $^{2}$ 
Table 1: Distribution of various microscopic lesions in placenta

\begin{tabular}{|c|c|c|c|c|}
\hline Microscopic lesion & $\begin{array}{l}\text { Study } \\
(n=75)\end{array}$ & $\begin{array}{c}\text { Group } \\
\%\end{array}$ & $\begin{array}{c}\text { Control } \\
(n=25)\end{array}$ & $\begin{array}{c}\text { Group } \\
\%\end{array}$ \\
\hline Inter villous fibrin deposition & 48 & $64 * * *$ & 3 & 12 \\
\hline Increased syncytial knotting & 48 & $64 * * *$ & 2 & 8 \\
\hline Stromal fibrosis & 42 & $56^{* * *}$ & 3 & 12 \\
\hline Cytotrophoblastic hyperplasia & 33 & $44 * * *$ & 0 & 0 \\
\hline Basement membrane thickening & 30 & $40 * * *$ & 0 & 0 \\
\hline Infraction (fig.3) & 21 & $28 * * *$ & 0 & 0 \\
\hline Fibromuscular sclerosis of fetal stem arteries & 21 & $28 * *$ & 1 & 4 \\
\hline Hypovascular villi & 24 & $32 * * *$ & 0 & 0 \\
\hline Fibrinoid necrosis of villi & 15 & $20 *$ & 1 & 4 \\
\hline Calcification & 27 & 36 & 8 & 32 \\
\hline Chronic villitis & 9 & $12 *$ & 2 & 8 \\
\hline
\end{tabular}

$P<0.05 \quad-$ Satisfactory signifi-cant

$P<0.01 \quad$ - Moderately signifi-cant

$P<0.001$ - Highly significant

Others - Insignificant

Placenta is the life line of the fetus and is a versatile organ with multiple functions, which are vital to the normal development of the fetus. Any disease adversely affecting the mother or the fetus brings about morphological changes in the placenta. These morphological changes can be studied by gross and microscopic examination of the placentas. The information obtained will be of great value in understanding the causes of adverse maternal, fetal and neonatal outcomes.

The present study was undertaken to assess the significance of placental changes in causing the reduction in birth weight of the babies in IUGR pregnancies.

\section{MATERIALS AND METHODS}

The present study was a retrospective and was conducted on 100 placentas collected in 2001 to 2002. Permission from the institutional review committee was obtained. Among these 75 placentas were from clinically diagnosed IUGR pregnancies and 25 were from normal pregnancies. Fetal birth weight was noted in all cases. Placentas were collected soon after delivery, washed with water so as to clean all blood. For fixation, the placenta was cut into the multiple longitudinal slices of $2 \mathrm{~cm}$ and kept in 10\% formalin for 2-3 days. After fixation, In addition to the sections from membranes, four whole thickness sections were taken from placenta. Additional sections were taken whenever gross lesions were detected along with the transverse section of the cord near to its attachment over the placental surface. Paraffin embedded blocks were made after processing and sections were cut approximately 2-3 microns thick and stained routinely by hematoxylin and eosin. Whenever needed, special stain viz. PAS, Masson's trichrome, Von Gieson were done to confirm villous pathology. All the data were collected from the medical reports.

\section{Statistics}

Statistical correlation of was carried out between them by using SPSS 18 version. Chi-square test with or without yate's correction was used as and when required. $\mathrm{P}<0.05$ was taken as critical level of significance.

\section{RESULTS}

Several major histologic changes occur in IUGR placentas compared to the normal placentas. These changes include intervillous fibrin deposition (64\% cases, fig. 1), increased syncytial knot-ting (64\% cases, fig. 2), stromal fibrosis (56\% cases), cytotrophoblastic hyperplasia ( $44 \%$ cases) and basement membrane thickening ( $40 \%$ cases).

In addition, hypovascular villi and infraction were present in $32 \%$ and $28 \%$ of placentas of IUGR pregnancies. All these changes were present in a lower number of cases of the control group $(\mathrm{p}<0.001)$. Fibromuscular sclerosis of fetal stem arteries was present in 28 percent of IUGR placentas $(\mathrm{P}<0.01)$. (Table 1)

Table 2 shows the association of fetal birth weight with the microscopic changes in placenta. For this purpose IUGR placentas are divided into four groups depending upon the number of microscopic findings. Group I placentas showed one significant microscopic change, and similarly

Group II to IV showed two, three and four or more microscopic features. The table shows that the number of microscopic changes increases with the decrease in birth weight, as 13 cases (61.9\%) out of 21 cases having birth weight less than $2 \mathrm{~kg}$ showed grade IV microscopic change. However when the birth weight is above $2 \mathrm{~kg}$ only $14.8 \%$ cases showed grade IV change. Since group I is having only one case, association was studied with group II and above. Thus there is statistically significant association between the birth weight and microscopic changes (chi square value 


\begin{tabular}{|c|c|c|c|c|c|c|}
\hline S.No & Fetal weight (Kgs) & Group I & Group II & Group III & Group IV & Total \\
\hline 1. & $2-2.4$ & 1 & 17 & 28 & 8 & 54 \\
\hline 2. & $1.5-2$ & 0 & 1 & 5 & 6 & 12 \\
\hline \multirow[t]{2}{*}{3.} & $<1.5$ & 0 & 0 & 2 & 7 & 9 \\
\hline & Total & 1 & 18 & 35 & 21 & 75 \\
\hline
\end{tabular}

Chi Square Value - 19.543

Degree of freedom $=4$

$P<0.005$

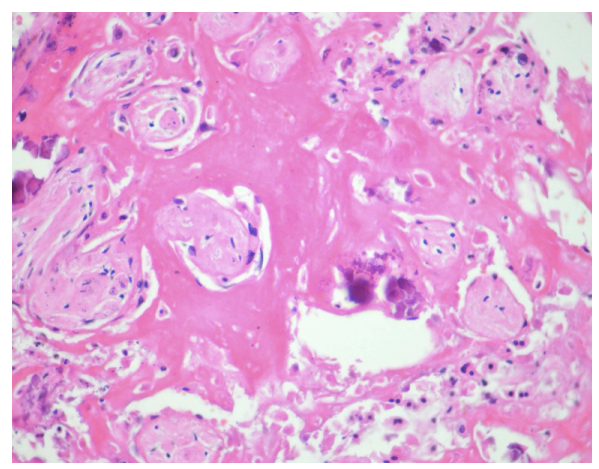

Figure 1: Photmicrograph showing placenta with massive intervillous fibrin deposition (HE stain, X40).

$=19.543$, Degree of freedom $=4, p<0.005$ ).

\section{DISCUSSION}

IUGR is associated with medical conditions that interfere with the circulation and efficiency of the placenta, with the development or growth of the fetus or with the general health and nutrition of the mother. IUGR may be a normal fetal response to nutritional or oxygen deprivation. Therefore, the issue is not the IUGR, but rather the ongoing risk of malnutrition or hypoxia. ${ }^{3}$ IUGR infants more frequently show multiple types of lesions in their placentas. Relationship of all placental lesions to IUGR was independent of each other. ${ }^{4}$ It is evident that there is not a single utero placental or villous lesion that result in fetal growth restriction. It is more likely that it is the accumulation (or total burden) of placental injury that when present for a sufficient time interval leads to IUGR. So, the patterns of lesions are more important in elucidating the casual pathways by which placental histopathology is translated into $\mathrm{IUGR}^{5}$ and histology examination of placenta may clarify a cause for delayed fetal growth. ${ }^{6}$ The severity of placental abnormalities expressed as cumulative number of placental lesions is a significant risk factor for IUGR. ${ }^{7}$ Redline et $\mathrm{al}^{8}$ demonstrated that five chronic patterns of placental injury are significantly increased in placentas from pregnancies complicated by IUGR. These are maternal and fetal vascular obstructive lesions, high grade villitis of unknown etiology (VUE), perivillous fibrinoid deposition and chronic abruption. In our study,

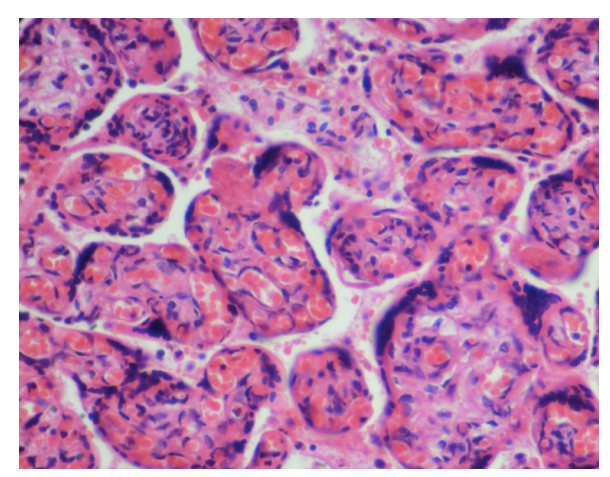

Figure 2: Photmicrograph showing placenta with increased syncytial knotting (HE stain, X40).

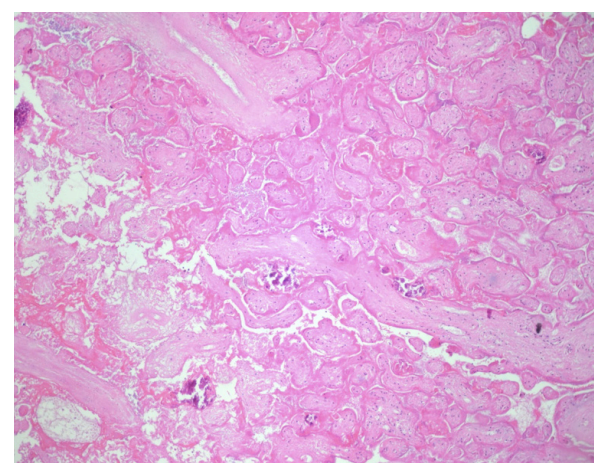

Figure 3: Photomicrograph showing pacental infarct with ghost like villi( $H \& E, 40 x)$

several, major histopathological changes were observed in IUGR placentas. They were intervillous fibrin deposition, increased syncytial knotting, stromal fibrosis of villi, cytotrophoblastic hyperplasia and thickening of basement membrane. In addition, infraction and hypovascular villi were present in a significant number of IUGR placentae. All these findings indicate disturbance in placental maturation and morphological changes due to reduced utero placental blood flow. End result of all these placental changes is a growth retarded fetus. Similar observations were made in studies on IUGR placental by Dawson L et al. ${ }^{9}$ Redline et $\mathrm{l}^{8}$ also observed increased intervillous fibrin deposition and extent of increased intervillous fibrin showed the strongest correlation with both placental weight and fetal weight. After comparing the microscopic change 
with the fetal birth weights, it is found that severity of IUGR is related to the severity of microscopic change in the placenta. Both number and severity of microscopic changes in placenta increased with decreasing fetal birth weight. The increasing number and severity of microscopic changes indicate the increasing burden on the placenta resulting in chronic placental insufficiency and the end result of which is a growth retarded fetus. Masodkar et al ${ }^{10}$ and Moriohio Iwata et $\mathrm{a}^{11}$ have found similar association between placental changes and birth weight. Thus placental histological examination may clarify the cause for delayed fetal growth and contribute to the better understanding of the genesis of intrauterine growth retardation. ${ }^{5}$

\section{CONCLUSION}

Pathological changes in placenta were higher amongst IUGR infants as compared to control group. Placental pathology examination gives more accurately the cause of IUGR infants. The findings suggest that chronic ischemia and associated secondary changes probably lead to improper perfusion and IUGR. Comparison of the microscopic changes with the fetal birth weight, revealed that severity of IUGR is related to the severity of microscopic change in the placenta. Therefore, to know the potential pathologies in the placenta, all placentas of IUGR infants should be examined.

\section{Conflict of interest: None}

\section{REFERENCES}

1. Park K. Textbook of Preventive and Social Medicine: 21 st ed. M/S Banarasidas Bhanot: Jabalpur; 2005. 560pp

2. Dutta DC. Text Book of Obstetric Including Perinatology and Contraception :7th ed. New Central Book Agency: Kolkata; 2010.467pp.

3. Kliegman RM, Behrman RE, Jenson HB, Stanton BF. Nelson Textbook of Paediatrics: 18th ed. Saunders: Philadelphia; 2007. 530pp. Crossref

4. Salafia CM. Vintzileous AM., Silberman L. Placental pathology of idiopathic intrauterine growth retardation at term. Am J Perinatol 1992;9:179-84. Crossref

5. Salafia CM. Placental pathology of fetal growth restriction, Clin. Obstet Gynecol 1997;40:740-49. Crossref

6. Rayburn W, Sander C, Corpaton A. Histologic examination of the placenta in the growth retarded fetus. Am J. perinatol 1989;6:58-61. Crossref

7. Vescardi RM, Sun CC. Placental lesion multiplication: risk factors for IUGR and neonatal cranial ultra sound abnormalities. Early Hum Dev 2001;62:1-10. Crossref

8. Redline RW. Placental pathology: A systematic approach with clinical correlations. Placenta. 2008;29:86-91. Crossref

9. Dawson L,Vijay Lakshmi S, Salham B. Histopathological changes of placenta in case of IUGR. India J Pathol Microbiol 1999.42:2167.

10. Masodkar AR, Kalamkar LR. Patki PS. Histopathology of placenta and correlation with fetal out-come. J Obstet. Gynaecol Ind 1985;35:294-6.

11. Iwata M, Matsuzaki N, Mitsuda N. Perinatal detection of ischaemic change in the placenta of the growth retarded fetus. J Ostet Gynaecol Ind 1993:82:494-9. 